\title{
Cardiovascular research days 2014: organ preservation and regeneration from basic science to clinic
}

Cortina d'Ampezzo, January 16-18, 2014

Published online: 8 January 2014

(C) Springer-Verlag Wien 2013

Thursday, 16th January 2014

\section{5:45 Introduction:}

A. Mazzucco (I)

G. Faggian (I)

\section{6:00 Symposium I: cellular preservation \\ Chair: A. Mazzucco (I), \\ G. Steinhoff (DE)}

\section{1}

Z. Papp (HU): Myocyte

\section{2}

S. Chlopizki (PL): Endothelium

\section{3}

K. Schmitt (DE): Neurons

\section{4}

G. Strapazzon (I): Cardiovascular and cellular responses to hypothermia, hypoxia and hypercapnia

\section{8:00 Coffee break}

18:30 Keynote lecture I:

Chair: G. Faggian (I),

B. K. Podesser (A)

\section{5}

T. M. Scarabelli (US): From cellular damage to organ protection

20:00 Welcome cocktail and dinner

Friday, 17th January 2014

\section{8:00 Symposium II: myocardial preservation \\ Chair: T. M. Scarabelli (US), \\ R. Seitelberger (A)}

\section{6}

B. K. Podesser (A): New aspects on protection of the old heart

\section{7}

J. Wojta (A): Role of inflammation in myocardial preservation 


\section{8}

D. Chambers (GB): Polarized arrest: an alternative to potassium-induced arrest

09

G. Szabo (DE): New drugs in the pipeline to protect the heart

\section{0:00 Coffee break}

\section{0:30 Symposium III: mini-oral session on selected abstracts (3 min talk + 3 min discussion) Chair: S. Hallström (A), B. H. Walpoth $(\mathrm{CH})$}

\section{0}

An isolated large animal heart platform for cardiac device development and research

\section{Granegger ${ }^{1,3}$, F. Moscato ${ }^{1,3}$, S. Mahr', J. Horvat ${ }^{1,2}$, P. Aigner ${ }^{1,3}$, D. Zimpfer ${ }^{2}$, H. Schima ${ }^{1,2,3}$ \\ ${ }^{1}$ Center for Medical Physics and Biomedical Engineering, Medical University of Vienna, Vienna, Austria ${ }^{2}$ Department of Cardiac Surgery, Medical University of Vienna, Vienna, Austria \\ ${ }^{3}$ Ludwig Boltzmann Cluster for Cardiovascular Research, Vienna, Austria}

Background: Although several small animal isolated heart setups are available, only a few groups developed a setting for isolated hearts from large animals capable of reproducing the pathophysiological hemodynamic conditions. Aim of this work was to establish a flexible isolated heart setup using pig hearts for development and testing of cardiovascular devices.

Methods: Pig hearts from the abattoir $(n=20)$ as well as from animal experiments were used $(n=10)$. The hearts were excised and connected to the isolated heart apparatus. After pressurecontrolled coronary reperfusion with blood the apparatus was switched to working-mode, which allowed left ventricular ejection into a Windkessel afterload. In 10 hearts a rotary blood pump (RBP) was implanted and the heart/pump hemodynamic interaction investigated.

Results: Hemodynamic conditions over a wide range were reproduced: cardiac output $(\mathrm{CO})$ was $0.6-9.4 \mathrm{~L} / \mathrm{min}$ at a preload of $15 \mathrm{mmHg}$ and an afterload of $70 \mathrm{mmHg}$, contractility could be adjusted from a dP/ $\mathrm{dt}_{\text {max }}$ of $629-3,625 \mathrm{mmHg} / \mathrm{s}$. Heart rate was in the range between 74 and $170 \mathrm{bpm}$. RBPs were successfully implanted in 10 hearts and the hemodynamics of the assisted circulation reproduced the ones observed in the clinic.

Conclusion: A large animal isolated heart setup was developed, which reproduced the human hemodynamics as well as the assisted circulation. By allowing isolated, defined and reversible changes of hemodynamic parameters, this tool does not only provide possibilities for device development but also offers the opportunity to perform pathophysiologic studies.

\section{1}

Local epicardial erythropoietin-plus-fibrin patch therapy for beneficial healing after myocardial infarction

\section{Klopsch, G. Kleiner, R. Gäbel, A. Skorska, M. Ludwig, P. Mela, S. Jockenhoevel, G. Steinhoff}

University of Rostock, 2 RWTH Aachen, Aachen, Germany

Background: High-dose intramyocardial Erythropoietin (EPO) therapy protects the myocardium from ischemic injury and promotes beneficial remodelling. We investigated the therapeutic efficacy of EPO at a moderate dose level and compared local and systemic delivery routes in a rat myocardial infarction (MI) model.

Methods: Following MI, EPO (300 U/ kg) was delivered immediately by intraperitoneal (EPO-S, $n=25)$ injection, intramyocardial (EPO-L, $n=23$ ) injection or epicardially as an EPOplus-fibrin patch (EPO-X, $n=26$ ). Groups were compared to each other and MI control groups with saline injections (MIC, $n=25$ ) or saline-plus-fibrin patch application (MIC-X, $n=29$ ). Heart functions were examined under baseline and Dobutamineinduced stress conditions using conductance catheter method 6 weeks after MI. Moreover, blood and myocardium were analysed including candidate genes and proteins at early $(24 \mathrm{~h})$ and late (6 weeks) stages after MI.

Results: Results illustrated superior cardiac functions and healing in EPO-X compared with all groups at 6 weeks after MI. Pressure-volume loops in EPO-X demonstrated $38 \%$ improved contractility (dpdtmax), $47 \%$ better elasticity (dpdtmin) and $15 \%$ enhanced ejection fraction compared with MIC-X. Furthermore, dpdtmax was 33 and $22 \%$ higher and dpdtmin was 32 and $23 \%$ greater in EPO-X compared with EPO-S and EPO-L, respectively. Infarction size, wall thinning, fibrosis and cardiomyocyte hypertrophy in EPO-X were reduced by $34,53,48$ and $22 \%$ compared mit MIC-X, respectively. Histological improvements in EPO-L and EPO-S were only moderate. Early real-time PCR analyses in EPO-X revealed exclusively elevated gene levels of intracardiac stem cell homing factors (SDF-1, CXCR-4, CD34), tissue-transformation factors (TGF-beta, MMP-2), anti-apoptotic Bcl-2 and cell cycle progression factor Cdc2. Blood analyses suggested, healing effects in EPO-X might have been hematopoiesis independent.

Conclusions: Immediate epicardial EPO-plus-fibrin patch better than systemic or local protein delivery restored cardiac performance after MI and early induced key molecules in myocardial regeneration. Therapeutic efficacy might be local-dose dependent.

\section{2}

Heart failure with preserved ejection fraction: $\mathrm{Ca}^{2+}$-mediated cardiomyocyte contractile dysfunction in a rat model with stable diastolic heart failure and renal impairment

\section{U. Primessnig ${ }^{1}$, B. Pieske ${ }^{1,2}$, F. Heinzel ${ }^{1,2}$}

${ }^{1}$ Department of Cardiology, Medical University Graz, Graz, Austria 
2Ludwig Boltzmann Institut for Translationale Heart Failure Research, Graz, Austria

Background: Heart failure with preserved ejection fraction (HFPEF) and renal impairment are often associated but the underlying pathomechanisms are largely unknown. We investigated tissue fibrosis and $\mathrm{Ca}^{2+}$-mediated cardiomyocyte contractile function in a rat model with HFPEF and compensated renal failure.

Methods: Seventy young male Wistar rats were subjected to subtotal nephrectomy (NXT) or sham operation (SOP). Serial blood/urine samples, echocardiography, pressure-volume loops were performed after 8 and 24 weeks. Left ventricular (LV) hypertrophy, fibrosis (picosirius-red-staining) and NCX protein expression (Western blot) were determined after sacrification. Cardiomyocyte function $\left(\mathrm{Ca}^{2+}\right.$ transients, cell shortening) was quantified in isolated cardiomyocytes without and with the NCX inhibitor SEA0400 (300 nM).

Results: NXT rats showed stable renal impairment with preserved urine excretion and elevated arterial pressure at 8 and 24 weeks. LV in NXT were significantly hypertrophied at 8 and 24 weeks but $\mathrm{LV}$ systolic function $(\mathrm{EF}, \mathrm{dP} / \mathrm{dt}$ ) were preserved. LVEDP, LA size and lung weight were significantly increased indicating HFPEF with pulmonary congestion. Fibrosis was increased in LV from NXT. LV cardiomyocytes showed significantly prolonged time for early (50\%) relaxation and decay of the $\mathrm{Ca}^{2+}$ transient. Time constant of the caffeine-induced $\mathrm{Ca}^{2+}$ transient (TAU) was significantly prolonged indicating reduced NCX forward mode activity, while NCX protein expression was upregulated. Acute treatment with SEA0400 (300 nM) significantly accelerated early relaxation in cardiomyocytes from NXT (8 weeks).

Conclusions: In this model of HFPEF cardiomyocyte relaxation was prolonged already at an early stage. Our results suggest increased NCX activity may contribute to contractile dysfunction. NCX represents a potential target to treat $\mathrm{Ca}^{2+}$-mediated cardiomyocyte contractile dysfunction in HFPEF.

\section{3}

\section{Myofilament carbonylation modulates human cardiomyocyte contractility}

\section{A. Balogh, A. Tóth, E. T. Pásztorné, L. Nagy, A. Kovács, J. Kalász, G. A. Contreras, I. Édes, Z. Papp}

Division of Clinical Physiology, Institute of Cardiology, University of Debrecen, Medical and Health Science Center, Debrecen, Hungary

Background: Oxidative stress is a common damaging factor in several cardiovascular disorders and conditions, e.g. in heart failure, in ischaemia-reperfusion injury or during cardiac surgery. The aim of this study was to investigate the effects of oxidative myofilament carbonylation in permeabilized human left ventricular cardiomyocytes.

Methods: In this study, protein carbonylation was monitored by the oxyblot method following in vitro applications of Fenton reaction constituents [iron, ascorbate and hydrogen-peroxide $\left.\left(\mathrm{H}_{2} \mathrm{O}_{2}\right)\right]$ known to produce hydroxyl radicals. Sulfhydryl group oxidation was assessed by the Ellman's assays in parallel. The $\mathrm{Ca}^{2+}$-activated active force, the $\mathrm{Ca}^{2+}$-independent passive force and the $\mathrm{Ca}^{2+}$-sensitivity of isometric force production (pCa50) were measured during force measurements in permeabilized cardiomyocytes before and after in vitro carbonylation.

Results: Carbonylation at the levels of several myofilament proteins (e.g. myosin heavy chain, $\alpha$-actinin, actin, myosin binding protein $\mathrm{C}$, desmin and myosin light chain 1) was enhanced with increasing concentration of $\mathrm{H}_{2} \mathrm{O}_{2}(0-105 \mu \mathrm{M})$. Carbonylation suspended active force generation following aggressive Fenton treatment $\left(105 \mu \mathrm{M} \mathrm{H}_{2} \mathrm{O}_{2}\right)$, whereas it decreased pCa50 (from $5.74 \pm 0.01$ to $5.65 \pm 0.01$; mean \pm SEM, $P<0.05$ ) and increased passive force (from $1.72 \pm 0.21 \mathrm{kN} / \mathrm{m}^{2}$ to $2.33 \pm 0.22 \mathrm{kN} / \mathrm{m}^{2}$ ) following the application of a submaximal $\left(3^{*} 103 \mu \mathrm{M}\right) \mathrm{H}_{2} \mathrm{O}_{2}$ concentration. None of these changes could be influenced by sulfhydryl group reduction.

Conclusion: Taken together, myofilament carbonylation dysregulates the contractile function in human cardiomyocytes, therefore, it may mediate the contractile dysfunction during oxidative stress. Eliminating the pathological effects of myofilament carbonylation could be a beneficial tool in preventing cardiomyocyte injury.

\section{4}

Inflammatory response in cardiac grafts after antithymocyte globulin preconditioning in the brain-dead donor

\section{J. Kremer ${ }^{1}$, G. K. Muschitz ${ }^{2}$, K. Aumayr ${ }^{3}$, K. Trescher ${ }^{1}$, D. Santer ${ }^{1}$, M. Kreibich'1, E. Dzlic ${ }^{1}$, A. O. Zuckermann' ${ }^{2}$, B. K. Podesser ${ }^{1}$}

${ }^{1}$ Ludwig Boltzmann Cluster for Cardiovascular Research, Vienna, Austria

${ }^{2}$ Department of Surgery, Allgemeines Krankenhaus, Vienna, Austria

${ }^{3}$ Department of Clinical Pathology, Allgemeines Krankenhaus, Vienna, Austria

Background: Hemodynamic and hormonal changes after brain death (BD) alter donor graft quality. Cardiac grafts present a higher risk for increased acute rejection and impaired graft survival. We investigated the influence of ATG preconditioning on immunoactivated donor hearts and its potential to reduce cytokine activation after BD.

Methods: Female OF-1 mice were divided 4 randomized groups ([BD-ATG], [BD-Control], [Therapy], [Control]). BD was induced with an intracranial balloon catheter, the mice were monitored for $6 \mathrm{~h}$ and the onset of BD was marked by an EEGflatline. Myocardial tissue sections were analyzed using ELISA and immunohistochemistry staining for IL-2 and IL-6.

Results: ATG pretreatment effected a significant reduction of IL-2 concentration in the myocardium after $\mathrm{BD}(p<0.05)$. IL- 6 levels tended to be lower in the BD-ATG groups. Less IL-6 in situ expression was found in the media tissue of coronary arteries in the ATG groups.

Conclusions: A reduction of IL-2 in cardiac grafts after ATG treatment in the BD group was found. Reduction of the inflammatory response in cardiac grafts can lead to improvement in organ quality by ATG preconditioning proceeding to transplantation. 
15

Influence of brain death on left ventricular pressure-volume relationship in a rat model of potential heart donor and its impact on posttransplant graft function

\section{S. Li' ${ }^{1}$, S. Korkmaz', S. Loganathan', T. Radovits ${ }^{2}$ P. Hegeds ${ }^{1,2}$, M. Karck' ${ }^{1}$, G. Szabó ${ }^{1}$ \\ 'Department of Cardiac Surgery, University of Heidelberg, Heidelberg, Germany \\ ${ }^{2}$ Heart Center, Semmelweis University, Budapest, Hungary}

Background: Heart transplantation became the most effective treatment for end-stage heart failure. Donors after brain death (BD) are currently the only reliable source for cardiac transplants. However, hemodynamic instability and cardiac dysfunction have been demonstrated in brain-dead donors and this could therefore also affect posttransplant graft function. Our aims were to evaluate the time-course cardiac influence of BD and its impact on graft left-ventricular $(\mathrm{LV})$ function in rats.

Methods: Lewis rats were either maintained BD for $5 \mathrm{~h}$ by inflation of a subdurally placed balloon catheter $(n=7)$ or subjected to sham-operation $(n=9)$. We assessed cardiac function during $5 \mathrm{~h}$. Then, hearts were excised, stored in cold preservation solution for $1 \mathrm{~h}$, and heterotopically transplanted. We evaluated graft function $1.5 \mathrm{~h}$ after transplantation.

Results: BD was associated with decreased LV contractility (ejection fraction: $37 \pm 6$ vs. $57 \pm 5 \%$; dP/dtmax: $4770 \pm 197$ vs. $7604 \pm 348 \mathrm{mmHg} / \mathrm{s}$; dP/dtmax-EDV: $60 \pm 7$ vs. $74 \pm 2 \mathrm{mmHg} / \mathrm{s}$; Emax: $\quad 2.4 \pm 0.1$ vs. $\quad 4.4 \pm 0.3 \mathrm{mmHg} / \mu \mathrm{l} ; \quad$ PRSW: $47 \pm 9$ vs. $78 \pm 3 \mathrm{mmHg} ; p<0.05)$, impaired ventricular relaxation $(\mathrm{dP} /$ dtmin: $-6,638 \pm 722$ vs. $-11,285 \pm 539 \mathrm{mmHg} / \mathrm{s}$; Tau: $12.6 \pm 0.7$ vs. $10.5 \pm 0.4 \mathrm{~ms} ; p<0.05)$, and increased end-diastolic stiffness (EDPVR: $0.33 \pm 0.14$ vs. $0.09 \pm 0.03 \mathrm{mmHg} / \mu \mathrm{l}, p<0.05$ ) $45 \mathrm{~min}$ after its initiation and for the rest of $5 \mathrm{~h}$ compared to controls. Moreover, after transplantation, graft systolic and diastolic functions were altered in the brain dead group compared to controls (reflected by decreased LV systolic and developed pressures, decreased $\mathrm{dP} / \mathrm{dtmax}$ and $\mathrm{dP} / \mathrm{dtmin}$, and prolonged Tau).

Conclusions: We have shown that BD is characterized with decreased systolic performance accompanied by impaired ventricular relaxation and increased end-diastolic stiffness within $45 \mathrm{~min}$ and maintained during $5 \mathrm{~h}$. Furthermore, BD increases the susceptibility of donor heart to ischemia/reperfusion injury after transplantation.

\section{6}

Neuroprotective effects of pharmacologically induced hypothermia with $\delta$-opioid receptor agonist DADLE and cannabinoid receptor agonist WIN55,212-2 during extracorporeal life support for cardiac arrest

\section{G. Tong', A. Rungatscher ${ }^{2}$, D. Linardi', A. Giacomazzi', M. Tessari², T. Menon², A. Mazzucco², G. Faggian², F. Berger ${ }^{1,3}$, K. R. Schmitt ${ }^{1}$}

${ }^{1}$ Congenital Heart Disease and Pediatric Cardiology, Deutsches Herzzentrum Berlin, Berlin Germany ${ }^{2}$ Department of Surgery, Division of Cardiac Surgery, University of Verona, Verona, Italy
${ }^{3}$ Pediatric Cardiology, Charité-Universitaetsmedizin, Berlin, Germany

Background: Extracorporeal life support (ECLS) and mild therapeutic hypothermia (TH) are regarded as effective therapeutic interventions for treating cardiogenic shock or cardiac arrest and post resuscitation care, respectively. However, the adverse neurological effects of therapeutic hypothermia have increasingly been recognized. Therefore, we investigated the neuroprotective effects of pharmacological induced hypothermia by $\delta$-opioid receptor agonist d-Ala2-d-Leu5 enkephalin (DADLE) and cannabinoid receptor agonist WINN55,212-2 in comparison to conventional mild therapeutic hypothermia after resuscitation from cardiac arrest in an ECLS rat model.

Methods: Ventricular fibrillation (VF) was induced in male Wistar rats for $10 \mathrm{~min}$, followed by venoarterial ECLS for $60 \mathrm{~min}$. Animals were randomized to four groups of ten: normothermia, mild hypothermia $\left(32^{\circ} \mathrm{C}\right)$, WIN55,212, and DADLE (both by intravenous infusions at $1 \mathrm{mg} / \mathrm{kg} / \mathrm{h}$ ) for the duration of ECLS. Brains were snap-frozen and stored at $-80^{\circ} \mathrm{C}$ until the hippocampus and cortex were extracted for analysis of IL-6, IL-10, MCP-1, iNOS, and RBM3 gene expression via qRT-PCR.

Results: We observed significant down-regulations of pro-inflammatory IL-6, MCP-1, and iNOS by mild TH and WINN55,212-2, but not by DADLE in comparison to normothermic control. Interestingly, anti-inflammatory IL-10 was significantly up regulated by mild $\mathrm{TH}$ and DADLE, but not WINN55,212-2, concurring with previous studies showing cannabinoid mediated down-regulation of IL-10. Finally, we observed significant up-regulation of RBM3 gene expression in all three treated groups, in comparison to normothermia.

Conclusion: Pharmacologically induced hypothermia by DADLE and WIN55,212-2 showed similar neuroprotective effects as mild therapeutic hypothermia in our ECLS rat model, albeit via different mechanisms. Additionally, the coinciding up-regulation of RBM3 by the pharmaceuticals with mild therapeutic hypothermia further support the implication of RBM3 as a potential effector for hypothermia-induced neuroprotection.

\section{7}

S-nitroso human serum albumin versus inhaled nitric oxide for the treatment of pulmonary hypertension in a chronic right ventricle volume overload model

\section{Linardi', A. Rungatscher', S. Hallström², E. Milani' ${ }^{1}$ M. Tessari ${ }^{1}$, GB. Luciani', A. Mazzucco', G. Faggian'1}

${ }^{1}$ Department of Surgery, Division of Cardiac Surgery, University of Verona, Verona, Italy ${ }^{2}$ Institute of Physiological Chemistry, Medical University of Graz, Graz, Austria

Background: The study aimed to compare the acute overall hemodynamic and oxidative stress effects of intravenous S-NOhuman serum albumin (S-NO-HSA) infusion and inhaled nitric oxide (iNO) in a chronic left-to-right shunt-induced pulmonary arterial hypertension model.

Methods: Male Wistar rats underwent surgical creation of aorto-caval fistula $(\mathrm{Qp} / \mathrm{Qs}>2.0)$. After 10 weeks they were randomly treated with human serum albumin (HSA) (controls; $n$. 25), S-NO-HSA ( $0.5 \mu \mathrm{mol} / \mathrm{kg} / \mathrm{h} ; \mathrm{n} .30)$ or iNO (20 ppm; n. 35) for $60 \mathrm{~min}$. Right ventricular contractility, right ventricular-vascular coupling and ventricular interdependence were assessed in vivo at different preloads by biventricular conductance catheters 
prior and after 60 min treatment. Heart and lung biopsies were obtained to determine oxidative stress by oxidized to reduced glutathione (GSSG/GSH) ratio and high-energy phosphates content.

Results: Both S-NO-HSA and iNO led to a significant reduction in right ventricular afterload expressed by effective pulmonary arterial elastance (Ea) (from $1.3 \pm 0.2$ to $0.5 \pm 0.3$ and $0.4 \pm 0.2$ respectively; $P<0.001)$. Only S-NO-HSA significantly improved right ventricle diastolic function (slope of end-diastolic pressurevolume relation) and contractility indicated by end-systolic elastance (Ees). Therefore a significant increase in the efficiency of ventricular-vascular coupling (Ees/Ea) occurred after S-NO-HSA but not iNO treatment (from $0.33 \pm 0.15$ to $0.98 \pm 0.21 ; P<0.005$ and from $0.35 \pm 0.16$ to $0.45 \pm 0.18$; $P<0.1$ respectively) with significant increase in left ventricular stroke volume ( $58 \pm 7$ vs. $18 \pm 9 \% ; P<0.003)$.

S-NO-HSA compared to iNO improved right ventricle phosphocreatine content $(27.08 \pm 11.35$ vs. $8.41 \pm 1.80 \mathrm{nmol} / \mathrm{mg}$ protein; $P<0.001)$ and myocardial energy charge $(0.85 \pm 0.03$ vs. $0.78 \pm 0.03 ; P<0.01)$. Both S-NO-HSA and iNO decreased lung and right ventricular GSSG/GSH ratio $(P<0.001)$.

Conclusion: S-NO-HSA is more effective than iNO in treating pulmonary hypertension, improving right ventricle diastolic function and right ventricular-arterial coupling with a positive effect on ventricular interdependence. This results in superior energetic reserve of the heart, despite similar reduction of lung and right ventricular oxidative stress.

\section{8}

Tenascin-C promotes ventricular hypertrophy and fibrosis during pressure overload in the mouse heart

\section{Kreibich ${ }^{1,2}$, E. Dzilic ${ }^{1,3}$, F. Nagel', D. Santer ${ }^{1,4}$, M. Krssak ${ }^{5}$, B. K. Podesser ${ }^{1,6}$, K. Trescher ${ }^{1,6}$}

${ }^{1}$ Ludwig Boltzmann Cluster for Cardiovascular Research, Vienna Working Heart Research Group, Vienna, Austria ${ }^{2}$ Heart Center Freiburg University, Department of Cardiovascular Surgery, Freiburg, Germany

${ }^{3}$ German Heart Centre Munich, Department of Cardiovascular Surgery, Munich, Germany

${ }^{4}$ General Hospital Hietzing, Department of Cardiovascular Surgery, Vienna, Austria

${ }^{5}$ Medical University of Vienna, Department of Medicine III, Division of Endocrinology and Metabolism, Vienna, Austria ${ }^{6}$ Regional Hospital St. Poelten, Department of Cardiac Surgery, St. Poelten, Austria

Introduction: The extracellular matrix protein Tenascin-C (TNC) influences cell adhesion and cardiac remodeling primarily after myocardial infarction (MI), but its role during ventricular hypertrophy remains unclear. A minimal invasive mouse aortic banding model was established to investigate the influence of TNC during left ventricular pressure overload (PO).

Methods: Following a partial sternotomy, aortic banding was performed (a ligature was placed between the innominate and the left common carotid artery) in TNC-knockout (TNC-KO) and wildtype (WT) mice: Sham surgery was performed (TNCKO-SHAM, WT-SHAM) respectively without tying the suture ( $n=6$ in every group). Histologic samples were collected after 1 week, 4 weeks, and 10 weeks. Also, hearts were assessed in vivo by cardiac magnetic resonance imaging after 10 weeks. Data are presented as mean \pm SEM.
Results: Hemodynamic function was significantly decreased only in the WT banding group: ejection fraction (WT: $48.06 \pm 4.48 \%$ vs. WT-SHAM: $62.20 \pm 1.98 \% p<0.01$; TNC-KO: $64.78 \pm 3.87 \%$ vs. TNC-KO-SHAM: $72.03 \pm 2.85 \%$ n.s.), cardiac output (WT: $43.68 \pm 4.23 \mathrm{ml} / \mathrm{g}$ vs. WT-SHAM: $82.80 \pm 4.68 \mathrm{ml} / \mathrm{g}$ $p<0.01$; TNC-KO: $67.18 \pm 7.45 \mathrm{ml} / \mathrm{g}$ vs. TNC-KO-SHAM: $83.94 \pm 6.52 \mathrm{ml} / \mathrm{g}$ n.s.). Also, septum thickness was significantly enlarged solely in operated WT mice (WT: $1.54 \pm 0.09 \mathrm{~mm}$ vs. WTSHAM: $1.11 \pm 0.03 \mathrm{~mm} p<0.01$; TNC-KO: $1.20 \pm 0.03 \mathrm{~mm}$ vs. TNCKO-SHAM: $1.04 \pm 0.03 \mathrm{~mm}$ n.s.). WT myocytes were significantly larger (WT: $521.49 \pm 25.7 \mu \mathrm{m}^{2}$ vs. WT-SHAM: $198.44 \pm 8.9 \mu \mathrm{m}^{2}$ $p<0.01$; WT vs. TNC-KO: $294.19 \pm 23.3 \mu \mathrm{m}^{2} p<0.01$ ) and hearts showed significantly more fibroses in the WT group (WT: $16.57 \pm 1.9 \%$ vs. WT-SHAM: $2.12 \pm 0.2 \% p<0.01$; WT vs. TNC-KO: $5.57 \pm 0.6 \% p<0.01)$ after 10 weeks.

Discussion: Hemodynamic function of TNC-null mice remained significantly less affected by ventricular remodeling during pressure overload. Also, there were only marginal signs of fibrosis in this group. Hence, TNC impacts ventricular remodeling not only after MI but also during PO.

\section{9}

PIGF-MMP9 engineered cardiomyocyte-derived iPS cells supported by PEG-fibrinogen hydrogel possess an enhanced capacity to repair the damaged myocardium

\section{Bearzi $i^{1,2}$, C. Gargioli ${ }^{2}$, G. Condorelli ${ }^{3}$, D. Seliktar ${ }^{4}$, R. $\operatorname{Rizzi}^{2,5}$}

${ }^{1}$ Institute of Genetic and Biomedical Research (IRGB-CNR), Milan, Italy

${ }^{2}$ IRCSS Multimedica, Milan, Italy

${ }^{3}$ Humanitas Clinical and Research Center, Rozzano (Milan), Italy

${ }^{4}$ Technion-Israel Institute of Technology, Technion City, Haifa, Israel

${ }^{5}$ Institute of Cellular Biology and Neurobiology, Rome, Italy

Background: Transplantation of cardiomyocytes (CM) with an appropriate biomaterial carrier for augmenting the efficacy of cell delivery to the heart may not be able to restore satisfactorily damaged myocardium.

Methods: To assess the enhanced cardiac regenerative capacity of CM-derived induced pluripotent stem cells (iPS), the cells were engineered for the expression of the angiogenic factor Placenta Growth Factor (PlGF-iPS, PiPS) and the Metalloproteinase 9 (MMP-9-iPS, MiPS) and delivered with an innovative hydrogel cell carrier. Injectable hydrogels were made from a polyethylene glycol-fibrinogen (PF) liquid precursor that was photo-polymerized in situ.

Results: The contractile properties of the combined PF-iPS implants were optimized in vitro and characterized evaluating cardiac biomarkers expression modulating the stiffness. The regenerative efficacy of the implants was then tested in vivo in an acute myocardial infarction (AMI) model using immunedeficient mice. The fractional shortening (FS) was drastically reduced in the saline control group 30 days after AMI $(17.1 \pm 2 \%)$, whereas the groups containing cells or PF showed a relatively slower time-dependent reduction of the FS (iPS + PF 1\% PEGDA, $32.3 \pm 3.5 \%$, MiPS + PF 1\% PEG-DA $31 \pm 3 \%$, PiPS + PF $1 \%$ PEG-DA $34 \pm 1 \%$ ) but the combination MiPS/PiPS was found to be the best approach $(37 \pm 1.8 \%)$.

Conclusion: This study demonstrates the enhanced efficacy in vivo of combined PF-MiPS-PiPS transplantation. 


\section{6:00 Symposium IV: poster session Chair: Z. Papp (HU), S. Chlopizki (PL)}

\section{0}

Hemodynamic energy of blood flow, endothelial effects and internal organs perfusion quality during short-term pulsatile and non-pulsatile circulatory support

\section{Dodonov', A. D. Milano', W. Van Oeveren W', B. Chiominto', Y. J. Gu², T. Menon'1, A. Carcereri De Prati $^{3}$, M. Tessari', G. Faggian', A. Mazzucco'}

${ }^{1}$ Cardiac surgery, Department of Surgery, University of Verona Medical School, Verona, Italy

${ }^{2}$ Cardiothoracic Surgery, University Medical Center of Groningen, Groningen, The Netherlands

${ }^{3}$ Biochemistry, Department of Life Science and Reproduction, University of Verona Medical School, Verona, Italy

Background: Although pulsatile circulation is natural for humans the real benefit of pulsatile blood pumps during shortterm and long-term circulatory support is still uncertain. The aim of the study was to evaluate a relationship between hemodynamic characteristics of pump-generated pulsatile blood flow, its endothelial effects and internal organs perfusion quality.

Methods: Thirty seven patients (age $80 \pm 3.3$ years) were prospectively randomized for heart surgery with either pulsatile (PP) or non-pulsatile (NP) perfusion performed by MEDOS DeltaStream centrifugal diagonal pump. Hemodynamic properties of blood flow were described in terms of Energy Equivalent Pressure (EEP) and Surplus Hemodynamic Energy (SHE). Systemic (SVRi) and pulmonary (PVRi) vascular resistance indices were monitored during operation and $18 \mathrm{~h}$ of postoperative period. Biomarkers of acute lung injury (ALI) - plasma Clara cell 16 protein (CC16), surfactant protein D (SP-D) and Elastase; acute liver/intestinal injury-plasma liver (L-FABP) and intestinal (I-FABP) fatty acid binding proteins; acute kidney injury (AKI) - urinary N-acetyl- $\beta$-D-glucosaminidase (NAG), kidney injury molecule-1 (KIM-1) and neutrophil gelatinase-associated lipocalin (NGAL), plasma ET-1 and erythrocyte E-NOS activity were assessed before surgery (T0), at sternum closure (T1), $2 \mathrm{~h}$ (T2) and $18 \mathrm{~h}$ (T3) of ICU-stay together with plasma lactates, free hemoglobin and perioperative creatinine clearance (CCr).

Results: PP was characterized by a significantly higher SHE transferred to the patients $(p<0.001)$, lower perioperative SVRi $(p<0.05)$, PVRi $(p<0.05)$ and lactates $(p<0.05)$. The SHE during PP showed direct and significant correlation with E-NOS activity $(\mathrm{R}=0.53)$ and free hemoglobin $(\mathrm{R}=0.58), p<0.01$; while the inverse correlation was found with ET-1 levels at $\mathrm{T} 1(\mathrm{R}=-0.44)$ and at $\mathrm{T} 2(\mathrm{R}=-0.45), p=0.03$. Plasma lactates during first postoperative hours correlated mostly with PVRi but not with SVRi, $p<0.001$. Mean SVRi calculated during NP paradoxically showed direct correlation with E-NOS activity $(\mathrm{R}=0.47, p<0.01)$ and inverse correlation with ET-1 levels $(\mathrm{R}=-0.54, p<0.01)$ detected at sternum closure. ALI markers, L-FABP and I-FABP did not show significant differences between groups, although PVRi at $\mathrm{T} 1$ correlated positively with CC16 at T1 $(\mathrm{R}=0.42, p=0.02)$ and with Elastase at $\mathrm{T} 1(\mathrm{R}=0.39, p=0.03)$. No difference was found between pre- and post-operative $\mathrm{CCr}$ in the PP-group (71 \pm 23 vs $60 \pm 35 \mathrm{~mL} / \mathrm{min}, p=0.27$ ), while it decreased significantly in the NP-group ( $67 \pm 24$ vs $45 \pm 15 \mathrm{~mL} / \mathrm{min}, p<0.001)$. The PPgroup showed significantly lowed urinary levels of NAG at T3 $(p=0.008)$, and NGAL at T1 $(p=0.01), \mathrm{T} 2(p=0.001)$ and T3 $(p=0.03)$ postoperatively.

Conclusions: PP produced by MEDOS DeltaStream DP3 centrifugal pump resulted in hemodynamic advantages and signs of better tissue perfusion when compared with NP. PP showed higher safety for renal physiology, resulting in better maintenance of glomerular filtration and lower renal tissue injury. Progressive systemic and pulmonary vasoconstriction observed during NP is supposed to depend mostly on the excessive sympathetic activation while endothelial-derived factors could serve to counterbalance this effect.

\section{1}

Will the myosin activator omecamtive mecarbil provide a potent therapeutic approach for systolic heart failure?

\section{Nagy, A. Tóth, Á. Kovács, B. Bódi, I. Édes, Z. Papp}

Division of Clinical Physiology, Institute of Cardiology, University of Debrecen, Medical and Health Science Center, Debrecen, Hungary

Background: Decreased cardiac contractility is a central feature of systolic heart failure, and omecamtive mecarbil, a novel cardiac myosin activator drug may provide inotropic support for weakened cardiac contractions. The aim of this study was to characterize a hypothetical $\mathrm{Ca}^{2+}$-sensitizing effect of omecamtive mecarbil and to determine the tissue selectivity of its action.

Methods: Direct force measurements were performed in myocyte-sized preparations derived from left ventricular or from diaphragmatic tissue samples of Wistar-Kyoto rats. Isolated and permeabilized cardiomyocytes/skeletal myofibers, attached between a force transducer and an electromagnetic motor were then exposed to test solutions with increasing concentrations of omecamtive mecarbil (between $10 \mathrm{nM}$ and $100 \mu \mathrm{M}$ ) to test its concentration dependent effects on $\mathrm{Ca}^{2+}$-regulated force production and on its $\mathrm{Ca}^{2+}$-sensitivity. Moreover, we made efforts to characterize drug induced changes in passive cardiomyocyte properties, as well as in the rate constant of the actin-myosin cross-bridge cycle (ktr).

Results: Administration of omecamtive mecarbil in the concentration range between 0.1 and $1 \mu \mathrm{M}$ led to significant increases $(P<0.05)$ in the $\mathrm{Ca}^{2+}$ sensitivity of force production in cardiomyocytes (pCa50 in drug free controls: $5.85 \pm 0.016$; and at 0.1 and $1 \mu \mathrm{M}$ drug concentrations: $5.98 \pm 0.014$ and $6.452 \pm 0.05$, respectively; $n=10$ ) as well as in diaphragmatic skeletal myofibers (pCa50 in drug free controls: $5.61 \pm 0.004$; and at $1 \mu \mathrm{M}$ drug concentration: $5.85 \pm 0.02 ; n=8$ ). A cardiotonic effect was also demonstrated in relaxing solutions $(\mathrm{pCa}=9.0)$, where surprisingly active force development was noticed at drug concentrations of $1 \mu \mathrm{M}$ and higher (Factive drug free: $0 \mathrm{kN} / \mathrm{m}^{2}$, and at $1 \mu \mathrm{M}$ drug concentration: $1.77 \pm 0.08 \mathrm{kN} / \mathrm{m}^{2} ; n=6$ ). Moreover, omecamtive mecarbil also significantly increased $\mathrm{Ca}^{2+}$-independent passive forces at $1 \mu \mathrm{M}$ concentration and higher in isolated cardiomyocytes (Fpassive drug free: $0.62 \pm 0.05 \mathrm{kN} / \mathrm{m}^{2}$ and at $1 \mu \mathrm{M}$ omecamtive mecarbil concentration: $2.12 \pm 0.12 \mathrm{kN} / \mathrm{m}^{2}$; $n=10$ ) and in diaphragmatic muscle fibers (Fpassive in drug free controls: $0.68 \pm 0.09 \mathrm{kN} / \mathrm{m}^{2}$, and at $1 \mu \mathrm{M}$ drug concentration: $0.93 \pm 0.08 \mathrm{kN} / \mathrm{m}^{2}, n=10$ ). Finally, omecamtive mecarbil slowed down the actin-myosin cycle as indicated by the significant decreases in the ktr parameter measured in isolated skeletal myofibers (ktr in drug free controls: $1.14 \pm 0.061 / \mathrm{s}$ and at $0.1 \mu \mathrm{M}$ drug concentration: $0.96 \pm 0.051 / \mathrm{s} ; n=8$ ) 
Conclusion: Our data illustrate omecamtive mecarbil as a potent positive inotropic agent, exerting its cardiotonic effect via a $\mathrm{Ca}^{2+}$-sensitizing mechanism. However, omecamtive mecarbil appears to be a non-selective myosin activator drug also enhancing the contractility of the rat diaphragm. Moreover, omecamtive mecarbil may also impair diastolic function through slowering of the actin-myosin cycle and by increasing cardiomyocyte passive force.

\section{2}

Investigating the vasodilating potency of two COS donators in an isolated mouse heart model

\section{P. Haller ${ }^{1}$, J. Krynicka', N. Nagel' ${ }^{1}$, D. Santer ${ }^{1}$, G. Brunhofer ${ }^{2}$, K. Trescher ${ }^{1}$, C. Studenik ${ }^{3}$, T. Erker ${ }^{2}$, B. K. Podesser ${ }^{1}$}

${ }^{1}$ Ludwig Boltzman Cluster for Cardiovascular Research, Vienna Working Heart Research Group, Vienna, Austria 2University of Vienna, Department of Medical Chemistry, Vienna, Austria

${ }^{3}$ University of Vienna, Department of Pharmacology and Toxicology, Vienna, Austria

Background: Drug induced vasodilatation is a promising therapeutic option for patients with cardiovascular disease. The aim of this study is to investigate the vasoactive potency of the carbonyl sulfide (COS) releasing substances MAH3HCL and SWS47HCL on coronary arteries in an isolated mouse heart model.

Methods: Hearts of adult male OF-1 mice were excised after heparinization and evaluated in a crystalloid perfused isolated Langendorff heart. Following 15' of baseline measurement, hearts were randomly perfused with the COS donator (MAH3HCL $n=6$, SWS47HCL $n=6$ ) or saline solution (control, $n=7$ and $n=7$ ) for 10'. Then recording of hemodynamics was continued for 50. Coronary flow (CF) and heart rate (HR) were monitored under constant afterload. Data are presented as mean \pm SEM compared to baseline.(recovery in \%)

Results: Whereas HR remained stable, MAH3HCL significantly increased CF with the onset of treatment (control: $98 \pm 1 \%$ vs. treatment: $123 \pm 6 \%, p<0.01$ ). When infusion was stopped CF normalized for the rest of the experiment. SWS47HCL instead showed no increase in CF compared to the control group (control: $96 \pm 10 \%$ vs. treatment: $93 \% \pm 7 \%$, n.s.). HR remained stable as well.

Conclusion: While SWS47HCL did not improve the CF Recovery, MAH3HCL significantly enhanced CF during administration and did not interact with heart rate. Further experiments are necessary to evaluate the efficacy of COS donors in the setting of coronary malperfusion.

\section{3}

The expression of TNC in the murine geriatric heart after myocardial infarction

\section{F. Nagel1', D. Santer, ${ }^{1,2}$, E. Dzilic ${ }^{1,3}$, M. Kreibich ${ }^{1,4}$, P. Haller ${ }^{1}$, M. Krssak ${ }^{5}$, B. K. Podesser ${ }^{1,6}$, K. Trescher ${ }^{1,6}$}

'Vienna Working Heart Research Group, Ludwig Boltzmann Cluster for Cardiovascular Research, Vienna, Austria ${ }^{2}$ Department of Cardiovascular Surgery, Hospital Hietzing, Vienna, Austria
${ }^{3}$ Department of Cardiac Surgery, German Heart Centre Munich, Munich, Germany

${ }^{4}$ Department of Cardiovascular Surgery, Heart Center

Freiburg University, Freiburg, Germany

${ }^{5}$ Centre of Excellence High Field MR, Department of

Radiology, Medical University of Vienna, Vienna, Austria

${ }^{6}$ Department of Cardiac Surgery, Landesklinikum St. Pölten,

St. Pölten, Austria

Background: Aging is associated with a higher incidence, mortality, and complication rate of myocardial infarction (MI). Tenascin-C (TNC) is a glycoprotein produced in the infarction border zone. Previous studies correlated high TNC expression with unfavorable outcome in patients with MI.

Methods: In male geriatric (OM, age: 18 months) and young (YM, age: 11 weeks) OF1 mice MI was induced by permanent LAD ligation. In SHAM groups the procedure was performed without LAD occlusion. 32 days after MI, cardiac magnetic resonance imaging (MRI) was used for hemodynamic evaluation. The expression of TNC 3, 7, and 32 days after MI was illustrated by immunohistochemistry and assessed by digital image analysis.

Results: In a 2-way ANOVA MRI examination showed significant effects of age and of MI vs. SHAM on ejection fraction (age: $p<0,001$; MI vs. SHAM: $p<0,001$ ), stroke volume heart weight ratio (age: $p<0,001$; MI vs. SHAM: $p<0,001$ ), cardiac output heart weight ratio (age: $p<0,05$; MI vs. SHAM: $p<0,05$ ), end-systolic (ESV; age: $p<0,01$; MI vs. SHAM: $p<0.001$ ), and end-diastolic left ventricular volumes (EDV; age: $p<0,05$; MI vs. SHAM: $p<0,001$ ). Moreover, MI had a significant effect on stroke volume (age: n.s.; MI vs. SHAM: $p<0,05)$. No significant effects of age and of MI vs. SHAM were found on heart rate and cardiac output. Furthermore, no significant interactions between the two factors were found in any parameter.

TNC expression peaked in both groups on the third day after MI. OM with MI showed an increased TNC expression 3, and 7 days after MI induction by tendency. In SHAM groups no specific TNC staining was detected.

Conclusion: We have successfully implemented a geriatric mouse model of MI with common signs of heart failure. Confirmed by MRI, we found significant hemodynamic differences between MI and SHAM groups, and also between OM and YM. Increased expression of Tenascin-C in geriatric hearts after MI may be a reason for impaired cardiac function. Further experiments are planned to proof these first results.

\section{4}

Expression of specific isoforms of L-type calcium channel (LTCC) $\beta$-subunits in human atrial cardiomyocytes impacts on calcium channels activity in atrial fibrillation

\section{Balycheva ${ }^{1,2}$, S. Schobesberger ${ }^{1}$, F. Schultz ${ }^{1}$, G. Faggian ${ }^{2}$, J. Gorelik ${ }^{1}$}

${ }^{1}$ Department of Cardiovascular Sciences, National Heart and Lung Institute, Imperial College London, UK

${ }^{2}$ University of Verona School of Medicine, Verona, Italy

Background: T-tubular invaginations (TTs) of the sarcolemma of cardiomyocytes contain junctional structures functionally coupling L-type calcium channels to the ryanodine receptors, and this configuration allows for calcium-induced calcium release and contraction. The integrity of TTs is crucial for maintaining calcium transient in atrial cardiomyocytes. On the 
other hand atrial fibrillation (AF) is associated with decreased L-type $\mathrm{Ca}^{2+}$ current (ICa) density and increased activity of single LTCCs. However, the nature of LTCC activity changes in AF remains unknown.

Objective: To determine the location, biophysical characteristics, and structural composition of functional L-type calcium channels (LTCCs) in human atrial cardiomyocytes.

Methods: LTCC main pore forming $\alpha 1 \mathrm{C}$ subunit and auxiliary $\beta$ subunits mRNA levels were determined by qPCR in right atrial appendage samples collected from patients with $\mathrm{AF}(\mathrm{AF}+)$ and normal rhythm (AF-). The levels of mRNA of $\alpha 1 \mathrm{C}, \beta 2$ and $\beta 4$ subunits decreased in atrial tissues from $\mathrm{AF}+$ patients. A novel technique of the super-resolution scanning patch-clamp was applied to identify functional LTCCs in TTs. LTCCs recorded in the TTs in atrial cardiomyocytes from $\mathrm{AF}+$ and $\mathrm{AF}-$ patients had similar current amplitude; however, open probability of LTCCs was 2.2-fold higher in cells isolated from $\mathrm{AF}+$ patients. Cardiomyocyte surface topography in isolated human atrial cardiomyocytes was characterized by scanning ion conductance microscopy (SICM) technique. Surface topography of cardiomyocytes appeared less regular with fewer Z-grooves $(0.37 \pm 0.06$ versus $0.56 \pm 0.02$ in $\mathrm{AF}+$ versus $\mathrm{AF}-$ cardiomyocytes, $P<0.001)$ in $\mathrm{AF}+$ patients as compared with AF- patients.) Both control cells and cells from $\mathrm{AF}+$ patients demonstrated heterogeneous surface topography independent on cell diameter.

Conclusion: Our study provides for the first time characteristics of functional LTCCs localised in the TTs in human atrial cardiomyocytes. We demonstrated significant degradation in surface topography and an increase in single LTCCs activity in atrial cardiomyocytes isolated from patients with $\mathrm{AF}$ which could underlie their arrhythmogenic phenotype.

\section{5}

Impact of donor organ quality on postoperative outcome in high urgency recipients-experience of an eurotransplant center

\section{B. Schmack', A. Weymann', A. Kazala', R. Arif', M. Farag', A. Doesch ${ }^{2}$, P. Raake ${ }^{2}$, S. Korkmaz', M. Karck' ${ }^{1}$, A. Ruhparwar', G. Szabo ${ }^{1}$}

${ }^{1}$ Department of Cardiac Surgery, University Hospital Heidelberg, Heidelberg, Germany

Department of Cardiology, University Hospital Heidelberg, Heidelberg, Germany

Objectives: The lack of suitable donors for heart transplantation (HTX) is increasing while at the same time the waiting list is increasing even greater. This fact leads on one side to high urgency (HU) listing ("sickest first line") of the recipients and on the other side to the need of acceptance of extended donor criteria resulting in allocation of donor hearts to mainly $\mathrm{HU}$ recipients in more than $90 \%$. The objective of our study was to investigate the impact of the donor profile and pre-implantation factors on postoperative outcome in $\mathrm{HU}$ recipients at our department representing an Eurotransplant center.

Methods: We retrospectively analyzed data of 50 consecutively performed HTX procedures within our institution in a 2-year period (July 2010 to August 2012). Clinical data were collected from medical records and outcome factors were calculated for pre-, intra- and postoperative factors. Survival was assessed by the Kaplan-Meier method. Main outcome parameters included survival, intensive care unit (ICU) stay, graft injury characterized by postoperative creatine kinase (CK; type MB)release and postoperative catecholamine usage.
Results: Of the 50 patients, $47(94 \%)$ were transplanted on HU status ( 35 male, $70 \%$ ), mean recipient age was $51.16 \pm 1.81$ years. 1-year survival was $84 \%$; overall survival was $80 \%$. Examined factors such as donor age ( $48.23 \pm 1.68$ years), donor age $\geq 55$ years $(n=19 ; 45.8 \%)$, donor organ ischemic time $(273 \pm 7.29 \mathrm{~min}$ ), donor gender (16 male; $32 \%$ ) and body surface donor/recipient index (BSA-d/r I; $1.01 \pm 0.15)$ as well as brain death-to-explant time (729.78 $\pm 42.88 \mathrm{~min})$, cause of brain death (non-traumatic intra-cranial bleeding $(n=26,52 \%)$, traumatic $(n=10,20 \%)$, apoplectic insult $(n=9,18 \%)$ and other $(n=5$, $10 \%)$ ) and post-operative CK-MB max release $(92.96 \pm 5.85 \mathrm{U} / \mathrm{l})$ did not show any association with the postoperative outcome measures. Early catecholamine therapy was mandatory in all cases. Therapy included a combination of nor-epinephrine and dobutamine in 27 (54\%), a triple therapy of epinephrine, norepinephrine and dobutamine in $10(20 \%)$, a combination of dobutamine and milrinone in $6(12 \%)$ and other combination in 7 ( $14 \%)$ patients. Mean ICU stay ( $7.18 \pm 1.31$ days), mean time on ventilator $(50.5 \pm 7.75 \mathrm{~h})$, catecholamine dosage and CK-MB max release $(92.96 \pm 5.85 \mathrm{U} / \mathrm{l})$ was not influenced by brain death-toexplant time, organ ischemic time, donor age or donor gender. However, prolonged ICU-stay following HTX was associated with a higher mortality $(p=0.021)$.

Conclusions: In our analysis, HU patients had a comparable survival with regular patients as reported by the ISHLT registry. However, in the special cohort donor related factors did not have an influence on the early and late postoperative outcome. Factors of success or failure of HTX are more likely to be depending on the recipient's condition rather then on the donor's side. Our data support the extended use of marginal donors in the high risk population, such as Eurotransplant HU recipients are. However, the need for discussion about the principles of organ allocation in HTX focusing the ideal recipient and the ideal time is more than crucial.

\section{6}

\section{$\mathrm{N}$-methyl D-aspartate receptor in human heart} function

\section{Kosenkov' ${ }^{1}$ A. Makhro' ${ }^{2}$ M. Tessari', M. Gassmann², A. Bogdanova ${ }^{2}$, G. Faggian'}

${ }^{1}$ Division of Cardiac Surgery, University of Verona Medical School, Verona, Italy

Institute of Veterinary Physiology, Vetsuisse Faculty and the Zurich Center for Integrative Human Physiology, University of Zurich, Zurich, Switzerland

Objectives: N-methyl D-aspartate receptors (NMDARs) are ionotropic glutamate receptors highly abundant in the brain and present in the lung, kidneys, red blood cells and in the heart of a variety of species including humans. Upon stimulation with glutamate and glycine these non-selective ion channels mediate calcium uptake and depolarize the membrane, both processes having crucial impact on heart function. Channels are heterotetramers consisting of glutamate-binding (NR2A, B,C or D) and glycine-binding (NR1, 3A and 3B) subunits. Subunit composition defines the agonist-, antagonist and ion permeability preferences, amplitude and deactivation time of the receptors. In rat heart NR2D and NR3A expression was recently shown to dominate. In human heart only NR1 was reported to be expressed so far. In this study we have explored the expression pattern of all known NMDAR subunits in four chambers of human heart and performed co-variance analysis of the transcript levels relat- 
ing them to the loss of cardiac rhythmicity and hypertrophic remodeling.

Methods: Thirty patients (age $67 \pm 11$ years) with mitral valve (MV) insufficiency underwent surgical treatment (MV repair was done in $80 \%$ of cases). Biopsy of myocardial tissue was performed during the cannulation for cardiopulmonary bypass procedure. Samples were obtained from all four heart chambers for assessment of the NMDAR subunit expression profile using real-time PCR. The standard 12-lead ECG and routine echocardiography were registered during preoperative evaluation of patients.

Results: The receptor subunit composition in human heart appears to be different from that of a rat. In human myocardium NR3A, NR2A, NR2B, NR2C and NR2D subunits are expressed in all four chambers. The higher expression of NR2B in left atrium (LA) was related to severity of hypertrophic remodeling in this chamber (dimensions). The transcript level for the NR3A subunit in LA showed a negative correlation with conductive and repolarization disorders revealed from the ECG analysis. Chronic atrial fibrillation was presented in $47 \%$ of patients and was associated with lower expression of NR2A and NR2C subunits in the right atrium.

Conclusion: The obtained data suggest that NMDARs may play an important role in control of heart function. This makes NMDARs into the attractive targets of pharmacological intervention.

\section{7}

Mild therapeutic hypothermia after hypoxia is neuroprotective possibly via upregulation of cold shock protein RBM3

\section{G. Tong ${ }^{1}$, L. M. Rosenthal' ${ }^{1}$, F. Berger ${ }^{1,2}$, K. R. Schmitt ${ }^{1}$}

${ }^{1}$ Congenital Heart Disease and Pediatric Cardiology, Deutsches Herzzentrum Berlin, Berlin, Germany ${ }^{2}$ Pediatric Cardiology, Charité-Universitaetsmedizin, Berlin, Germany

Objective: Hypoxic-ischemic insult after cardiac arrest or resuscitation can lead to a hypoxic state in brain tissue, thus resulting in neurological sequelae and long term dysfunction. Mild to moderate therapeutic hypothermia (TH) is known to be an effective cytoprotectant, and therefore possibly improving the neurological outcome of these patients. TH is not only protective through slowing cellular metabolism and reducing the oxygen demand, but can further alter inflammation, apoptosis, and cellsurvival pathways. However, the underlying mechanisms of THinduced neuroprotection remains to be elucidated. Cold shock proteins, specifically, the RNA-binding motif protein 3 (RBM3), have been observed to be upregulated in response to hypothermia and recently implicated in TH-induced neuroprotection. Therefore, we investigated the effects of mild TH after hypoxic injury on cell death, mitochondrial function, cell proliferation, and RBM3 regulation in the human neuroblastoma SK-N-SH cell line.

Methods: SK-N-SH neuroblastoma cells were exposed to hypoxic conditions $\left(0.2 \% \mathrm{O}_{2}, 5 \% \mathrm{CO}_{2}\right)$ at $37^{\circ} \mathrm{C}$ in a fully humidified anoxic chamber for $24 \mathrm{~h}$. Following hypoxic insult, normoxic conditions $\left(20 \% \mathrm{O}_{2}, 5 \% \mathrm{CO}_{2}\right)$ at either normothermia $\left(37^{\circ} \mathrm{C}\right)$ or mild hypothermia $\left(33.5^{\circ} \mathrm{C}\right)$ for up to $96 \mathrm{~h}$. Cell viability was accessed by LDH and NSE releases, mitochondrial activity was measured by MTT reduction and intracellular ATP content, cell proliferation was evaluated by BrdU incorporation, and RBM3 regulation was quantified by qRT-PCR and Western Blot.
Results: Mild therapeutic hypothermia markedly rescued SK-N-SH neuroblastoma cells from hypoxia-induced injury as observed in significant decreases in LDH and NSE releases, compared to normothermic controls. Interestingly, no differences in cell proliferation in response to either hypoxia or hypothermia were observed. Mitochondrial reductase activity was significantly decreased by both hypoxia and hypothermia, however the combination of hypoxia and subsequent hypothermia resulted in the largest decrease in mitochondrial activity. We also observed significant decreases in cellular ATP levels in un-cooled hypoxia injured SK-N-SH cells, which was successfully attenuated by mild TH for 24 and $48 \mathrm{~h}$. Additionally, RBM3 expressions, both mRNA and protein, were significantly up-regulated by mild $\mathrm{TH}$, as well as the combination of hypoxia and hypothermia. However, hypoxia alone did not induce RBM3 expression in our experimental settings with the SK-N-SH cell line.

Conclusion: Moderate therapeutic hypothermia following hypoxic insult reduces neuronal cell death. RBM3 up-regulation in response to mild hypothermia could be one possible mechanism for hypothermia-induced neuroprotection.

\section{8}

Pharmacologically induced hypothermia with cannabinoid receptor agonist WIN55,212-2 vs mild therapeutic hypothermia in a rat model of cardiac arrest with extracorporeal life support

\section{E. Milani', A. Rungatscher', D. Linardi', A. Giacomazzi', M. Tessari', T. Menon'1, S. Hallström², A. Mazzucco', G. Faggian ${ }^{1}$}

${ }^{1}$ Department of Surgery. Division of Cardiac Surgery, University of Verona, Verona, Italy

${ }^{2}$ Institute of Physiological Chemistry, Medical University of Graz, Graz, Austria

Background: To compare the hemodynamic, cardioprotective and metabolic effects of cannabinoid receptor agonist WIN55,212-2 with mild therapeutic hypothermia and normothermic control on post-resuscitation myocardial function in a rat model of extracorporeal life support (ECLS).

Methods: Ventricular fibrillation (VF) was induced in male Wistar rats. After 10 min of untreated VF, venoarterial ECLS was instituted for $60 \mathrm{~min}$. At the beginning of ECLS animals were randomized to three groups of ten: normothermia, hypothermia $\left(32^{\circ} \mathrm{C}\right)$ and WIN55,212 intravenous infusion $(1 \mathrm{mg} / \mathrm{kg} / \mathrm{h})$. Cooling to $32^{\circ} \mathrm{C}$ or normothermia or drug infusion lasted for the entire ECLS. Plasma samples and myocardial biopsies were obtained and left-ventricular (LV) function was assessed by a conductance catheter at baseline and after weaning from ECLS.

Results: WIN55,212-2 administration produced pharmacologic hypothermia $\left(32-34^{\circ} \mathrm{C}\right)$ and led to a significantly better recovery of the slope of the LV end-systolic pressure volume relationship (Ees) and Preload recruitable stroke work (PRSW) than hypothermia and normothermia: as percent of baseline $87+12$ vs. $54+9$ vs. $39+11$ and $88+11$ vs. $59+10$ vs. $33+8$ respectively $(p<0.01)$. LV stiffness expressed by end-diastolic pressure volume relationship (EDPVR) was significantly lower after WIN55,212-2 administration $(p<0.05)$. LV relaxation described by Tau was preserved after WIN55,212-2 treatment but not after hypothermia or normothermia $(p<0.01)$. WIN55,212-2 and not hypothermia significantly increased phosphorylation of the kinase ERK1 and $2(2.8+0.4$ and $2.5+0.3$ vs. $0.5+0.2$ and $0.4+0.1$-fold of baseline levels) $(p<0.01)$. Both WIN55,212-2 and hypothermia but not normothermia increased phosphorylation of Akt. This resulted 
in a reduced cardiomyocyte apoptotic index (TUNEL, $p<0.01$ ). WIN55,212-2 but not hypothermia nor normothermia preserved high energy phosphates content and energy charge and reduced lactate plasma concentration.

Conclusions: Pharmacologically induced hypothermia with WIN55,212-2 was more effective than mild therapeutic hypothermia in recovering myocardial function after ECLS and in improving myocardial protection through activation of the pro-survival kinases Akt and ERK1/2.

\section{9}

Effects of polarizing microplegia vs standard high-potassium depolarizing microplegia on intracellular metabolism, pro-survival kinases, and apoptosis in humans

\section{K. Gandhi', A. Rungatscher ${ }^{1}$, S. Hallström ${ }^{2}$, M. Tessari', D. Linardi' ${ }^{1}$ E. Milani', J. Vinten-Johansen ${ }^{3}$, G. P. Dobson ${ }^{4}$, F. Onorati ${ }^{1}$, A. Mazzucco', G. Faggian'}

${ }^{1}$ Division of Cardiac Surgery, University of Verona, Verona, Italy

2Institute of Physiological Chemistry, Medical University of Graz, Graz, Austria

${ }^{3}$ Cardiothoracic Research Laboratory, Department of Surgery

(Cardiothoracic), Emory University, Atlanta, Georgia, USA

${ }^{4}$ Heart Research Laboratory, Dept of Physiology and

Pharmacology, James Cook University, Queensland, Australia

Background: Potassium-enriched depolarizing arrest (DA) is still the worldwide accepted method for myocardial protection. Despite animal models have demonstrated superior protection with no-potassium polarizing arrest (PA), and clinical studies reported lower perioperative myocardial enzymatic release, intra-cellular effects of PA vs DA in human myocardial specimens are still ill-defined.

Methods: Sixty consecutive patients undergoing isolated aortic valve replacement were randomly allocated to adenosinelidocaine-magnesium blood PA (30 patients) or standard 4:1 blood DA (30 patients) according to "Buckberg-protocol". Coronary sinus blood was sampled for lactate release preoperatively (T0) and after reperfusion (T1). Myocardial specimens from right atrium were analyzed for high-energy phosphate content, energy charge, activation of pro-survival kinases Akt and ERK1/2, and cardiomyocyte apoptosis (TUNEL-assay) at T0 vs T1. Spontaneous recovery of sinus rhythm (SRSR) at aortic declamping was also recorded.

Results: Blood lactate from coronary sinus was lower at $\mathrm{T} 1$ after PA $(2.04 \pm 0.03 \mathrm{mmol} / \mathrm{L}$ vs $2.57 \pm 0.02$ after DA; $p=0.03)$, whereas SRSR was higher ( $64 \%$ vs $32 \%$ in DA-patients; $p=0.02$ ). Plasma $\mathrm{K}^{+}$did not significantly changed at T1 in PA patients ( $p=$ NS vs T0). PA, not DA, preserved myocardial high-energy phosphate content and energy charge $(0.79 \pm 0.02$ vs $0.73 \pm 0.02$; $p<0.001)$. Activation of pro-survival kinases Akt and ERK1/2 at T1 was higher after PA, not after DA $(\Delta \mathrm{pAkt} / \mathrm{Akt}-0.26$ vs 0.85 ; $\Delta$ pERK1/ERK1 -0.18 vs $0.77 ; \Delta$ pERK2/ERK2 -0.28 vs 0.65 ; $p<0.001$ after PA, $p=$ N.S. after DA). Cardiomyocyte apoptotic index was lower after PA $(0.13+0.10$ vs $0.35+0.12 ; p=0.01)$.

Conclusion: PA preserves myocardial high-energy phosphates and energy charge, and activates pro-survival kinases Akt and ERK resulting in attenuated apoptosis. PA is superior to DA at the myocellular level.

\section{0}

Generation of iPSC-based cardiac model systems for investigating mechanisms of dilated cardiomyopathy due to nuclear lamina defects

\section{E. Di Pasquale ${ }^{1,2}$, H. Nakahama², M. Miragoli 2,3 , A. Forni ${ }^{4}$, R. Roncarati $i^{1,5}$, P. Carullo, ${ }^{1,2}$, P. Kunderfranco ${ }^{2}$, L. Papa ${ }^{5}$, G. Faggian", G. Condorelli, ${ }^{1,2}$}

${ }^{1}$ Institute of Genetic and Biomedical Research, UOS of Milan, National Research Council of Italy, Rome, Italy

${ }^{2}$ Humanitas Clinical and Research Center, Rozzano, Italy

${ }^{3}$ Department of Bioscience, Center of Excellence for Toxicological Research INAIL exISPESL, University of Parma, Parma, Italy

${ }^{4}$ Division of Cardiac Surgery, University of Verona, Verona, Italy

${ }^{5}$ IRCCS, Multimedica Institute, Milan, Italy

Background: Dilated cardiomyopathy (DCM) is a disease of the heart muscle. It represents the third most common cause of heart failure and the most frequent cause of heart transplantation in the Western world. Causes may be various and about $25-50 \%$ of cases are familial. More than 40 genes, mostly encoding components of the nucleo-cytoskeletal apparatus, have been linked to DCM. Among these, mutations in genes encoding Lamin A/C are the most common. Lamins are expressed in all somatic cells and control many cellular processes such as the maintenance of the nuclear stucture, transcriptional regulation and chromatin remodelling. They are also crucial for physically coupling the nucleus and the cytoskeleton. Despite this knowledge, the role of Lamin A/C in the heart and the consequences of their alteration in DCM need to be further elucidated. With the advent of induced pluripotent stem cells (iPSC), the generation of human disease-specific cardiomyocytes (CMs) in vitro has become a viable option and offers the opportunity to create cellular models, in which molecular pathways leading to a given disease may be investigated. Here, we employed human CMs generated from iPSC derived from a Lamin-defective DCM family: the main aim was to comprehend the role of the nuclear lamina defect that lead to functional and molecular dysfunctions in DCM patients in the context of human CMs. the functional and molecular dysfunctions due to nuclear lamina defects in the context of human CMs.

Methods: iPSC were generated from skin fibroblasts by a lentiviral method using STEMCCA excisable polycistronic vectors, encoding the four "pluripotency" factors (Oct-4, Sox-2, Klf-4 and c-Myc); CMs have been differentiated from them by the embryoid bodies method. Molecular investigations included gene expression studies (Realtime PCR, Western blot, Illumina arrays), confocal microscopy for sub-cellular localization and sarcomere organization detection. Electrophysiological properties of CMs have been determined using scanning ion conductance microscopy.

Results: In this study, we generated CMs from iPSC derived from patients in a DCM family carrying a point mutation (K219T) in the gene encoding Lamin A/C proteins, and from a sub-group within the family with an additional variant (L4855F) in the gene encoding the "giant" sarcomeric component Titin, segregating with a more severe phenotype. As expected, both patients' fibroblasts and CMs exhibited reduced, disorganized nuclearmembrane Lamin A/C expression and nuclei enlargement and envelope invaginations. DCM-CMs also showed deregulation of expression of genes mainly involved in transcriptional regulation and DNA synthesis, indicating a key role of Lamin A/C in chromatin remodeling and regulation of transcription. Compared to 
control healthy subjects in the same family, CMs derived from the DCM patients exhibited an abnormal sarcomeric organization, with a higher percentage of abnormal cells in the double mutants compared to the single mutants ( 70 vs $50 \%$ ). Furthermore, analysis of calcium handling revealed an increased susceptibility of mutant cells to b-adrenergic stimulation by isoproterenol. The mechanisms underlying the observed phenotype are still under investigation.

Conclusions: Even under preliminary investigation, our data obtained here indicate that iPSC-derived CMs may represent a good model to investigate functional and molecular phenotypes of DCM and can potentially shed light on the function of the nuclear lamina in the heart function and pathophysiology. Such a model may also be useful in the future for screening for novel pharmacological therapies.

\section{7:00 Symposium V: preservation of organs functions Chair: G. Szabo (DE), L. Gottin (I)}

\section{1}

G. Condorelli (I): MicroRNA in cardiovascular diseases

\section{2}

A. Rajek (A): Intraoperative options for acutely failing hearts

\section{8:00 Coffee break}

\section{8:30 Keynote lecture II:}

Chair: G. Faggian (I),

B. K. Podesser (A)

\section{3}

A. Zuckermann (A): 30 years of experience with the surgical therapy of end-stage heart failure

\section{0:00 Social Dinner}

Saturday, 18th January 2014

\section{9:00 Symposium Vl: regenerative medicine for CVD \\ Chair: G. Condorelli (I), B. H. Walpoth $(\mathrm{CH})$}

\section{4}

K. Guan (DE): Patient-specific induced pluripotent stem cells for heart regeneration

\section{5}

G. Steinhoff (DE): Development of new techniques in cardiac regeneration

\section{0:00 Coffee break}

\section{0:30 Symposium VII: organ replacement \\ Chair: A. Zuckermann (A), \\ G. Steinhoff (DE)}

\section{6}

G. Faggian (I): Bridge-to-bridge, bridge-totransplant or destination therapy?

\section{7}

B. H. Walpoth $(\mathrm{CH})$ : Future of vascular grafts

11:30 "Carl Apstein Award" and
closing remarks
G. Faggian (I), B. K. Podesser (A),
B. H. Walpoth $(\mathrm{CH})$




\section{Author index}

A

Aigner, P., 10

Arif, R., 25

Aumayr, K., 14

B

Balogh, A., 13

Balycheva, M., 24

Bearzi, C., 19

Berger, F., 16, 27

Bódi, B., 21

Bogdanova, A., 26

Brunhofer, G., 22

\section{C}

Carcereri De Prati, A., 20

Carullo, P., 30

Chambers, D., 8

Chiominto, B., 20

Chlopizki, S., 2

Condorelli, G., 19, 30, 31

Contreras, G.A., 13

\section{D}

Di Pasquale, E., 30

Dobson, G.P., 29

Dodonov, M., 20

Doesch, A., 25

Dzilic, E., 18, 23

Dzlic, E., 14

\section{E}

Édes, I., 13, 21

Erker, T., 22

F

Faggian, G., 16, 17, 20, 24, 26, 28-30, 36

Farag, M., 25

Forni, A., 30

\section{G}

Gäbel, R., 11

Gandhi, K., 29

Gargioli, C., 19

Gassmann, M., 26

Giacomazzi, A., 16, 28

Gorelik, J., 24

Granegger, M., 10

$\mathrm{Gu}$, Y.J., 20

Guan, K., 34

\section{$\mathrm{H}$}

Haller, P., 22, 23

Hallström, S., 17, 28, 29
Hegeds, P., 15

Heinzel, F., 12

Horvat, J., 10

$J$

Jockenhoevel, S., 11

\section{K}

Kalász, J., 13

Karck, M., 15, 25

Kazala, A., 25

Kleiner, G., 11

Klopsch, C., 11

Korkmaz, S., 15, 25

Kosenkov, D., 26

Kovács, A., 13, 21

Kreibich, M., 14, 18, 23

Kremer, J., 14

Krssak, M., 18, 23

Krynicka, J., 22

Kunderfranco, P., 30

L

Li, S., 15

Linardi, D., 16, 17, 28, 29

Loganathan, S., 15

Luciani, G.B., 17

Ludwig, M., 11

M

Mahr, S., 10

Makhro, A., 26

Mazzucco, A., 16, 17, 20, 28, 29

Mela, P., 11

Menon, T., 16, 20, 28

Milani, E., 17, 28, 29

Milano, A.D., 20

Miragoli, M., 30

Moscato, F., 10

Muschitz, G.K., 14

\section{N}

Nagel, F., 18, 23

Nagel, N., 22

Nagy, L., 13, 21

Nakahama, H., 30

O

Onorati, F., 29

P

Papa, L., 30

Papp, Z., 1, 13, 21

Pásztorné, E.T., 13
Pieske, B., 12

Podesser, B.K., 6, 14, 18, 22, 23

Primessnig, U., 12

R

Raake, P., 25

Radovits, T., 15

Rajek, A., 32

Rizzi, R., 19

Roncarati, R., 30

Rosenthal, L.M., 27

Ruhparwar, A., 25

Rungatscher, A., 16, 17, 28, 29

$\mathrm{S}$

Schobesberger, S., 24

Santer, D., 14, 18, 22, 23

Scarabelli,T.M., 5

Schima, H., 10

Schmack, B., 25

Schmitt, K., 3

Schmitt, K.R., 16, 27

Schultz, F., 24

Seliktar, D., 19

Skorska, A., 11

Steinhoff, G., 11, 35

Strapazzon, G., 4

Studenik, C., 22

Szabo, G., 9, 15, 25

\section{$\mathrm{T}$}

Tessari, M., 16, 17, 20, 26, 28, 29

Tong, G., 16, 27

Tóth, A., 13, 21

Trescher, K., 14, 18, 22, 23

V

van Oeveren, W., 20

Vinten-Johansen, J., 29

W

Walpoth, B.H., 37

Weymann, A., 25

Wojta, J., 7

Z

Zimpfer, D., 10

Zuckermann, A., 33

Zuckermann, A.O., 14 